\title{
In Situ Determination of Local Ce Oxidation States During Redox Reactions
}

\author{
Renu Sharma and Peter Crozier
}

Center for Solid State Science, Arizona State University, Tempe, AZ 85287-1704

Three way catalysts (TWC) are used to reduce the common pollutants $\mathrm{CO}, \mathrm{NO}_{\mathrm{x}}$, and unburned hydrocarbons escaping from the exhaust system of automobiles. The effectiveness of the catalyst is directly related to the oxygen storage capacity (OSC) capacity of the material. $\mathrm{CeO}_{2}$ based catalysts are commonly used in catalytic converters because Ce can exist in both +3 and +4 oxidation states and the oxides possess high oxygen mobility at low temperature $\left(\approx 300^{\circ} \mathrm{C}\right)$ resulting in an easy oxidation-reduction (redox) cycles. The ceria/zirconia mixed oxide system $\left(\mathrm{Ce}_{1-\mathrm{x}} \mathrm{Zr}_{\mathrm{x}} \mathrm{O}_{2}\right)$ has been found to have higher OSC than the pure oxides and consequently are the material of choice for many TWC applications. ${ }^{1}$ Here we outline a novel approach for performing in situ observations on the nanochemistry of Ce based catalysts during catalysis. Using an environmental transmission electron microscope (ETEM) (a modified Philips 430 TEM operating at $300 \mathrm{KV}$ ) we perform in situ reduction in 2 Torr of $\mathrm{H}_{2}$. The local cation valence state was determined by electron energy-loss spectroscopy (EELS) during redox reactions using a Gatan Imaging Filter (GIF). The EELS approach is relatively simple and can be used to determine the oxidation state of each cation in mixed oxides for both ordered and disordered systems.

Large crystals (2-5 micron) of $\mathrm{CeO}_{2}$ were pressed into a $3 \mathrm{~mm}$ diameter pellet that was polished, dimpled and ion milled to obtain electron transparent regions. Various mixed oxide catalysts, containing $\mathrm{CeO}_{2}$ as one component, were loaded on Mo grids. These samples were heated using a furnace-heating holder in 2- 2.5 Torr of dry $\mathrm{H}_{2}$ gas during observation. A single crystal oriented in $<110>$ crystallographic direction (Figure 1a), was observed to form a superlattice at $800^{\circ} \mathrm{C}$ in 2 Torr of $\mathrm{H}_{2}$ gas due to reduction (Figure 1b). Energy-loss spectra, from small areas of the sample approximately $0.5 \mu \mathrm{m}$ in diameter, recorded at room temperature and at $700^{\circ} \mathrm{C}$ in $\mathrm{H}_{2}$ are shown in Figure 1c and 1d respectively. Both spectra show the $\mathrm{O} \mathrm{K}$-edge at $530 \mathrm{eV}$ and the $\mathrm{Ce}_{45}$ edge at $879 \mathrm{eV}$. Apart from the change in $\mathrm{O} / \mathrm{Ce}$ intensity ratio, the relative intensity of $\mathrm{Ce}_{45}$ white-lines was also observed to change during heating in $\mathrm{H}_{2}$. The $\mathrm{O} / \mathrm{Ce}$ signal ratio was measured from the energy-loss spectra using standard methods (Gatan EL/P program). We assumed that the largest $\mathrm{O} / \mathrm{Ce}$ signal ratio corresponded to an $\mathrm{O} / \mathrm{Ce}$ concentration ratio of 2 and a Ce oxidation state of +4 . We use the experimentally obtained oxidation state data to calibrate the Ce white-line intensities. The oxidation state of ceria recorded under different reducing conditions is plotted as a function of the corresponding $\mathrm{M}_{5} / \mathrm{M}_{4}$ white-line intensity ratio (Figure 1e). An inverse power law gives a reasonable fit to the data of Figure 1e and the oxidation state, A, can be expressed in terms of the white-line ratio, $\mathrm{R}$, through

$$
\mathrm{A}=1.81 \mathrm{R}^{-1.627}
$$

This permits us to directly determine the oxidation state of $\mathrm{Ce}$ in more complex mixed oxide samples simply by measuring the $\mathrm{M}_{5} / \mathrm{M}_{4}$ ratio $(\mathrm{R})$. A heating and cooling cycle was performed on the commercial $\mathrm{Ce}_{0.5} \mathrm{Zr}_{0.5} \mathrm{O}_{2}$ mixed oxide catalysts in order to study redox behavior in situ. The Ce oxidation state determined from the white-lines is plotted as a function of temperature (Figure 1f). The initial average oxidation state of this material is about +3.7 suggesting that the starting material is already partially reduced. The onset of further reduction occurs between 400 and $450^{\circ} \mathrm{C}$ and by $550^{\circ} \mathrm{C}$ the oxidation state drops to around +2.9 . We find that the mixed oxide sample is much easier to reduce than HSA pure ceria sample and oxidizes during cooling (Figure 1f). Even though the 
sample was cooled in an $\mathrm{H}_{2}$ atmosphere the ceria still re-oxidized back to the original +3.7 state by $400^{\circ} \mathrm{C}$. The oxygen to re-oxidize the sample is presumable obtained from the residual background gases (base pressure $\sim 10^{-3}$ Torr). We find that the re-oxidation process shows evidence for a hysterisis effect.

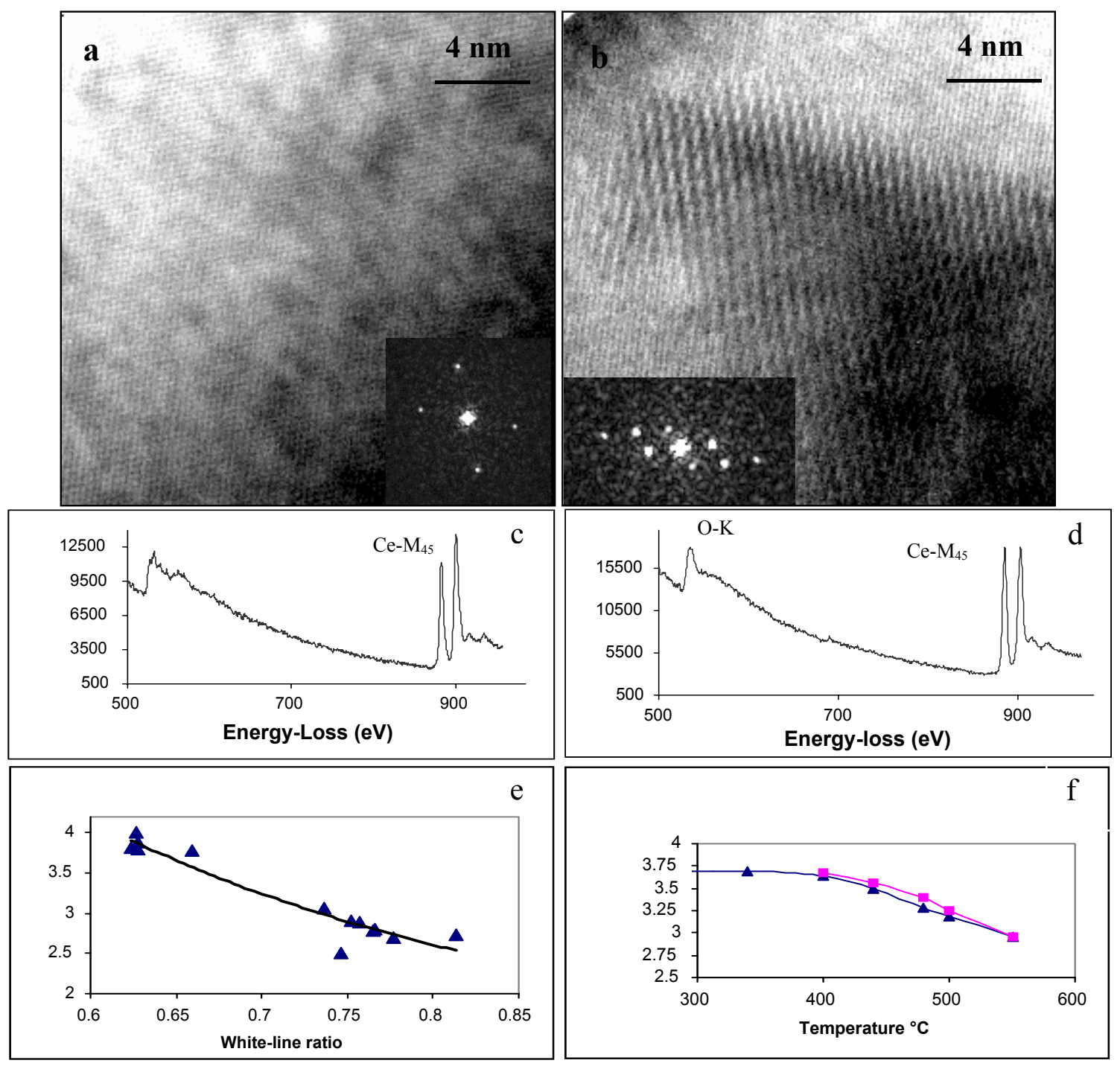

Figure 1(a) HREM image of a single crystal $\mathrm{CeO}_{2}$ oriented along $<110>$ zone axis with diffractogram inset in lower right corner; (b) same region at $800^{\circ} \mathrm{C}$ in 2 Torr of dry $\mathrm{H}_{2}$ after heating for 25 minutes. The diffractogram (inset in lower left corner) confirms the superlattice observed in HREM image. Typical EELS data obtained (c) at room temperature and (d) at $700^{\circ} \mathrm{C}$ in $\mathrm{H}_{2}$ showing the change in white-line $\left(\mathrm{Ce}_{45}\right)$ ratio. (e) Ce oxidation state plotted as a function of white-line intensity. (f) Oxidation state as a function of temperature in $\mathrm{Ce}_{0.5} \mathrm{Zr}_{0.5} \mathrm{O}_{2}$ during heating (triangles) and cooling (squares).

\section{References}

1. Fornasiero, P., Kasper, J., and Graziani, M., 1997, J. of Catalysis, 167, 576.

2. Dr. Kang and Center for High Resolution Electron Microscopy are gratefully acknowledged. 